\title{
Evaluation of students' acceptance of the leap motion hand gesture application in teaching biochemistry
}

\begin{abstract}
This paper presents an early stage of the Leap Motion controller regarding user acceptance in the teaching and learning process. The Leap Motion is a new device for a hand-gesturecontrolled user interface. For appropriate evaluation, a novel experiment and questionnaire were created utilizing 35 Biochemistry undergraduate students in Enzymology from the Universiti Putra Malaysia. The subjects participated in the user experiment and performed several tasks, such as rotating, translating and zooming in and out on the molecules. The tasks were performed using the Molecules application on an Airspace platform. The research compared the performance of Leap Motion with mouse interaction. As a result, $79.2 \%$ of the respondents gave a positive opinion about the Leap Motion because of its ease of use, acceptance, effectiveness and accuracy. These students were excited and looked forward to implementing the Leap Motion in class. Thus, the Leap Motion controller can potentially be used as a teaching tool for a better learning experience of the biomolecule.
\end{abstract}

Keyword: Leap motion; Molecules; Evaluation; Hand gesture; Biochemistry 